Urol. Prax. 2020 · 22:105-110

https://doi.org/10.1007/s41973-020-00110-1

Publié en ligne: 28 . Septembre 2020

(c) The Author(s) 2020

\section{Introduction}

Les tumeurs malignes du pénis sont rares et leur incidence varie selon les régions du monde. Elle atteint $1 / 100 ` 000$ hommes dans l'union européenne et aux Etats-Unis mais est toutefois plus fréquente dans certaines régions, représentant 1-2\% des tumeurs de l'homme en Amérique du Sud, en Asie du SudEst et dans certaines régions de l'Afrique. L'incidence du cancer de la verge augmente avec lâge avec un pic à 60 ans [1].

Le cancer de la verge est fréquent dans les zones à haute prévalence d'infection au papillomavirus humain (HPV), ce qui peut notamment expliquer les variations géographiques de son incidence. En effet, une infection à HPV est retrouvée dans 70 à $100 \%$ des lésions pré-cancéreuses de la verge PeIN (i.e. penile intra-epithelial neoplasia), et dans 30 à $40 \%$ des cas de cancer [2]. Le mécanisme d'action du HPV dans la carcinogénèse nest pas tout à fait établi, mais il est présumé qu'il aurait des interactions avec des oncogènes et des gènes suppresseurs de tumeur. Les sous-types les plus fréquent associés au cancer de la verge sont le HPV-16 et HPV-18. Toutefois, il est à noter que la survie spécifique au cancer à 5 ans est significativement meilleure dans les tumeurs HPV-positives [3]. Concernant la vaccination, cette dernière est recommandée et remboursée en Suisse depuis 2016, mais on ne trouve à ce jour pas de

Supervision: Astrid Roosendaal (médecin cheffe de clinique), Service d'urologie, Hôpitaux Universitaires de Genève, Genève 14, Suisse. Professeur Christophe Iselin (médecin chef de service), Service d'urologie, Hôpitaux Universitaires de Genève, Genève 14, Suisse

\title{
Elisabeth Grobet-Jeandin
}

Service d'urologie, Hôpitaux Universitaires de Genève, Genève, Suisse

\section{Recommandations sur la prise en charge du cancer du pénis}

recommandation européenne à ce sujet [4].

Ainsi, le principal facteur de risque identifié pour développer un cancer de la verge est une infection à HPV, dont la traduction clinique est notamment le condylome acuminé. Parmi les autres facteurs de risques, on peut retenir le phimosis qui entraîne une macération et un défaut d'hygiène préputial à l'origine $\mathrm{du}$ cancer de la verge. Certaines études démontrent à ce sujet que la circoncision néo-natale a un caractère préventif, mais il n'en est pas de même lorsqu'elle est pratiquée à l'âge adulte. Également, une inflammation chronique du gland, par exemple une balano-posthite ou un lichen scléro-atrophique, représente un facteur de risque du cancer du pénis.

Certains traitements dermatologiques comme la photothérapie UVA ou les psoralènes ont été associés au développement du cancer de la verge. Le tabagisme a un rôle non négligeable dans la carcinogenèse avec un risque augmenté de 3 à 5 fois par rapport aux patients non-fumeurs [5].

Enfin, le risque d'avoir un cancer de la verge est augmenté également dans les populations de bas niveau socio-économique, comme dans les populations rurales, chez les patients avec des partenaires sexuels multiples, un statut célibataire actif sexuellement, ou des premiers rapports sexuels précoces.

Tous ces éléments sont ainsi à rechercher à l'anamnèse lors de la prise en charge d'un patient avec suspicion de tumeur pénienne, afin d'identifier précocement les patients à risque, et de leur proposer des mesures préventives.

\section{Histo-pathologie}

Concernant les lésions de la verge, il convient de différencier les lésions à risque, les lésions pré-cancéreuses, les lésions cancéreuses pré-invasives et le carcinome épidermoïde.

Les lésions à risque sont des lésions bénignes avec un potentiel d'évolution cancéreuse, comme le lichen scléro-atrophique, les condylomes vénériens (sans PeIN) et la papulose bowénoïde.

Les lésions pré-cancéreuses sont quant à elles le PeIN, le CIS (PeIN3), lérythroplasie de Queyrat et la maladie de Bowen. Les lésions cancéreuses préinvasives sont le condylome acuminé géant (tumeur de Buschke-Löwenstein) et la maladie de Paget extra-mammaire. Leur potentiel malin est plus important et un traitement précoce est ainsi nécessaire afin de prévenir leur extension loco-régionale.

Le carcinome épidermoïde représente $>95 \%$ des cancers péniens. Il en existe différents sous-types avec un pronostic variable. Du point de vue histo-pathologique, il est subdivisé en grades basés sur la différenciation cellulaire selon la World Health Organisation (WHO). Il existe ainsi quatre grades comme suit:

- G1:bien différencié

- G2: modérément différencié

- G3:peu différencié

- G4: indifférencié

Le grade histopathologique doit absolument figurer dans le rapport anatomopathologique car il a un impact primordial sur la prise en charge ( $c f$. infra).

La classification TNM 2016 (Tumor, Node, Metastasis) est celle utilisée dans la pratique courante pour déterminer le stade pathologique du carcinome de la verge. 
Tab. 1 Classification TNM (Tumor, Node, Metastasis) 2016 du cancer de la verge

\begin{tabular}{ll}
\hline T & Tumeur primitive \\
Tis & Carcinome in situ \\
Ta & Carcinome verruqueux non infiltrant \\
T1 & $\begin{array}{l}\text { Tumeur envahissant le tissu conjonc- } \\
\text { tif sous-épithélial }\end{array}$
\end{tabular}

T1a Sans invasion lympho-vasculaire ou périneurale, de grade 1 ou 2

T1b Avec invasion lympho-vasculaire ou périneurale, de grade 3 ou 4

T2 Tumeur envahissant le corps spongieux, avec ou sans atteinte de l'urètre

T3 Tumeur envahissant le corps caverneux, avec ou sans atteinte de l'urètre

T4 Tumeur envahissant d'autres structures adjacentes

$N \quad$ Ganglions régionaux

cN0 Absence de ganglions régionaux inguinaux palpables ou visibles

cN1 Palpation d'un ganglion inguinal unilatéral mobile

cN2 Palpation de ganglions multiples ou bilatéraux mobiles

cN3 Ganglions inguinaux fixés ou ganglions pelviens, unilatéraux ou bilatéraux

pNO Absence de ganglions régionaux inguinaux

pN1 Métastase ganglionnaire dans un ou deux ganglions inguinaux

pN2 Métastase ganglionnaire dans $>2$ ganglions inguinaux unilatéraux, ou atteinte bilatérale

pN3 Métastase ganglionnaire pelvienne, extension extra-capsulaire

M Métastases à distance

M0 Absence de métastase à distance

M1 Présente de métastase à distance

Cette classification est résumée dans le

- Tab. 1 et illustrée dans la • Fig. 1.

Sur la base de la classification TNM et du grade tumoral, on distingue trois groupes à risque:

- Tumeurs à faible risque ( $\leq \mathrm{pT} 1 \mathrm{G} 1)$

- Tumeurs de risque intermédiaire (pT1 G2)

- Tumeurs à haut risque (pT1G3, pT2-3 G1-3)

\section{Diagnostic}

Une anamnèse complète à la recherche des facteurs de risques doit être systéma- tique. Elle doit également se concentrer sur la temporalité des symptômes, la présence de troubles mictionnels concomitants et la sexualité du patient.

Sur le plan clinique, le signe principal est une lésion indurée du pénis, qui est par ailleurs distale dans $95 \%$ des cas. Afin d'optimiser la prise en charge chirurgicale ultérieure, il convient de décrire précisément son aspect, sa localisation, sa taille, la présence d'une éventuelle extension locale, par exemple au niveau urétral, caverneux ou périnéal. Il est essentiel d'examiner le patient en position décalottée, car une lésion cancéreuse peut se trouver cachée sous un phimosis. Au statut, il est également primordial de recherche les adénopathies inguinales. La présence de ces dernières aura un impact sur le bilan d'extension préopératoire et sur le diagnostic.

Concernant les examens complémentaires, il n'existe à ce jour pas de marqueur biologique permettant le diagnostic du cancer du pénis. Le bilan précéde une éventuelle prise en charge chirurgicale est ainsi bioptique et/ou radiologique.

Léchographie ne représente pas l'examen de référence pour le diagnostic du cancer de la verge. Si effectuée, elle doit être réalisée après injection d'un agent érectile et permet d'évaluer l'infiltration des corps caverneux. L'IRM a une meilleure sensibilité pour évaluer l'extension locale, urétrale et caverneuse. Elle est également réalisée après une érection pharmaco-induite et permet de préciser le stade clinique en cas de doute lors du statut. Elle n'est toutefois pas indispensable avant la prise en charge chirurgicale si le statut est évident. Le rôle principal de l'IRM est de différencier les stades cT2 et cT3, ce qui peut parfois être difficile à l'examen clinique.

Une biopsie de la lésion permet l'obtention d'un diagnostic anatomopathologique et est recommandée en cas de lésion de la verge. Une biopsieexérèse est généralement préférée à une biopsie millimétrique. Cette dernière est souvent réalisée en cabinet d'urologie ambulatoirement, sous anesthésie locale.

Comme mentionné ci-dessus, la palpation des aires ganglionnaires inguinales est essentielle dans le diagnostic clinique du cancer de la verge, puisquelle va dicter la suite du bilan préopératoire et de la prise en charge (cf. ๑ Fig. 2) :

- En l'absence d'adénopathie inguinale palpable et en présence d'une tumeur à bas risque, aucun examen supplémentaire n'est nécessaire. Ceci est à pondérer selon le BMI du patient, la palpation ganglionnaire étant souvent plus difficile chez les patients obèses. En l'absence d'adénopathie inguinale palpable mais avec une tumeur à risque intermédiaire et haut, la recherche d'un ganglion sentinelle sera à effectuer au bloc opératoire pour déterminer la prise en charge (cf. infra).

- En présence d'adénopathie inguinale palpable, on peut éventuellement réaliser une cytoponction à l'aiguille fine pour obtenir un diagnostic histo-pathologique. Ce résultat n'aura toutefois de valeur qu'en cas de positivité. L'absence de tumeur à la cytoponction à l'aiguille fine ne permettant pas d'exclure une invasion ganglionnaire. L'examen complémentaire de choix dans ces circonstances est alors le PETCT au 18F-FDG qui permet de réaliser un bilan d'extension complet. Alternativement, un CT abdominopelvien permet d'évaluer l'atteinte ganglionnaire et métastatique.

- En cas d'atteinte ganglionnaire confirmée $(\mathrm{cN}+)$, il conviendra de réaliser une radiographie standard thoracique ainsi qu'un CT abdominopelvien. Alternativement, on peut réaliser une PET-CT au 18F-FDG. En cas de suspicion clinique d'atteinte osseuse, une scintigraphie osseuse peut également être envisagée.

Le ganglion sentinelle représente le premier relais lymphatique dans lequel on peut trouver des métastases ganglionnaires. Comme dans la prise en charge du cancer du sein, la recherche du ganglion sentinelle au bloc opératoire joue un grand rôle dans le traitement du cancer de la verge. Elle se fait dans le même temps opératoire que l'excision chirurgicale de la tumeur primaire. La procédure consiste en l'injection péri-tumorale de Technetium $99 \mathrm{~m}$ la veille de l'intervention ou le jour-même. Lors de la chirurgie, on 
Résumé · Zusammenfassung

recherche le ganglion à lâge d'une sonde de détection gamma: cette technique permet de retrouver le ganglion sentinelle dans $97 \%$ des cas, et ainsi d'éviter une lymphadénectomie étendue dans $76 \%$ des cas. Ceci représente un grand avantage pour les patients atteints de cancer de la verge vu la morbidité relativement haute liée à la lymphadénectomie ( $c f$. in$f r a)$. En cas de ganglion sentinelle positif, on effectuera un curage ganglionnaire modifié uniquement du côté du ganglion sentinelle. En l'absence de ganglion visualisé, on peut répéter la procédure, ou alternativement réaliser un curage modifié bilatéral.

\section{Traitements}

Les traitements conservateurs locaux sont plutôt réservés aux lésions précancéreuses ou cancéreuses superficielles, ou dans les situations où une chirurgie n'est pas envisageable. Dans tous les cas, il convient de réaliser une biopsie de la lésion et d'y associer une circoncision. Les traitements locaux peuvent consister en l'application de crème cytotoxique (p.e. imiquimod $5 \% 3 \mathrm{x} /$ semaine pendant 4 à 6 semaines ou 5 -FU $2 \mathrm{x} / \mathrm{J}$ pendant 2 à 6 semaines). Une lasérisation au Laser Yag ou au $\mathrm{CO}_{2}$ est une alternative. Enfin, la photothérapie dynamique après application d'un topique photo-sensibilisant (p.e. aminole'vulinate) est également une solution. On obtient une réponse complète dans environ 50 à $60 \%$ des cas et une surveillance rapprochée tous les trois mois est donc nécessaire. En cas d'échec, un second traitement topique ou laser n'est pas recommandé.

Le traitement chirurgical par excision est le traitement de choix du carcinome épidermoïde de la verge. Il convient dopter pour la chirurgie la plus conservatrice possible. Tout comme pour les traitements topiques, une circoncision doit être systématiquement associée. Au bloc opératoire, il est recommandé de réaliser un examen extemporané des marges chirurgicales en cas de chirurgie conservatrice. En effet, lobtention d'une marge chirurgicale négative est un impératif absolu. La limite de la marge de sécurité est débattue dans la littérature, mais

Urol. Prax. 2020 -22:105-110 https://doi.org/10.1007/s41973-020-00110-1

(c) The Author(s) 2020

\section{E. Grobet-Jeandin}

\section{Recommandations sur la prise en charge du cancer du pénis}

\section{Résumé}

Le cancer du pénis est une pathologie relativement rare dans nos régions qui est souvent associée à une infection à HPV. II se manifeste par une lésion de la verge, distale dans la majorité des cas. L'examen clinique local est essentiel pour réaliser le staging local, déterminant pour le bilan préopératoire. Le statut ganglionnaire inguinal doit également être systématique, étant donné que l'atteinte ganglionnaire est le facteur pronostic principal de la maladie. Le traitement du cancer du pénis étant essentiellement chirurgical, il convient d'adresser tout patient présentant une lésion suspecte de tumeur de la verge à un urologue, pour que ce dernier réalise une biopsie avant d'envisager le traitement. Par la suite, la chirurgie sera le plus conservatrice possible. L'obtention de marges de résections négatives est impérative. Il existe plusieurs techniques de curage ganglionnaire (curage inguinal modifié, lymphadénectomie inguinale totale) qui sont appliquées selon le risque et le stade TNM (tumor, node, metastasis).

Le suivi post-opératoire est essentiellement clinique et comprend l'auto-examination régulière de la verge ainsi que des ganglions inguinaux, en association avec des visites médicales régulières jusqu'à cinq ans postopératoires.

\section{Mots clés}

Tumeur de la verge $\cdot$ Stade TNM - HPV . Ganglion sentinelle $\cdot$ Curage ganglionnaire

\section{Empfehlungen zur Behandlung des Peniskarzinoms}

\section{Zusammenfassung}

Das Peniskarzinom ist in unseren Regionen eine relativ seltene Erkrankung, die häufig mit einer HPV(Humane Papillomviren)-Infektion assoziiert ist. Die Erkrankung manifestiert sich durch eine meistens distale Penisläsion. Die klinische Lokaluntersuchung ist wesentlich für das lokale Staging, das für die präoperative Beurteilung von entscheidender Bedeutung ist. Der inguinale Lymphknotenstatus muss ebenfalls systematisch ermittelt werden, da der Lymphknotenbefall bei dieser Erkrankung der wichtigste Prognosefaktor ist.

Die Therapie bei Peniskarzinom ist vor allem chirurgisch. Daher sollte jeder Patient mit tumorverdächtiger Penisläsion für eine Biopsie zu einem Urologen überwiesen werden, bevor die Therapie überlegt wird. Der Eingriff sollte dann so organerhaltend wie möglich gestaltet werden, wobei unbedingt darauf zu achten ist, dass die Schnittränder frei von Tumorgewebe sind. Es stehen je nach Risiko und TNM-Stadium verschiedene Techniken zur Lymphknotenentfernung zur Verfügung (modifizierte inguinale Lymphadenektomie, radikale Lymphadenektomie).

Die postoperative Nachsorge ist vor allem klinisch und beinhaltet regelmäßige Selbstuntersuchungen des Penis und der inguinalen Lymphknoten sowie regelmäßige Arztbesuche bis zu fünf Jahre nach dem Eingriff.

\section{Schlüsselwörter}

Peniskarzinom · Peniskrebs · TNM-Stadium . HPV · Sentinel-Lymphknoten · Lymphknotenentfernung - Lymphadenektomie on estime globalement qu'une marge de 3-5 mm est suffisante.

La technique chirurgicale n'est pas standardisée: elle dépend des possibilités techniques, selon taille et localisation de la tumeur. Toutefois, en cas d'amputation pénienne partielle avec reconstruction urétrale et méatale, il est essentiel de laisser une longueur de pénis restant de $3 \mathrm{~cm}$ minimum afin de permettre une miction dirigée. En cas d'amputation totale de la verge, il conviendra de réaliser une périnéostomie. Enfin, en cas d'émasculation complète, un traitement de substitution hormonale de testostérone sera à introduire en post-opératoire à vie.

La radiothérapie peut être une alternative de prise en charge de la tumeur primaire en cas de tumeur inférieure à $4 \mathrm{~cm}$. Elle consiste en une curiethérapie interstitielle par fils d'iridium 192 à une dose de $>60$ Grays. Elle doit être associée systématiquement à une circoncision au préalable. Elle se complique toutefois possiblement d'une sténose du méat, de 


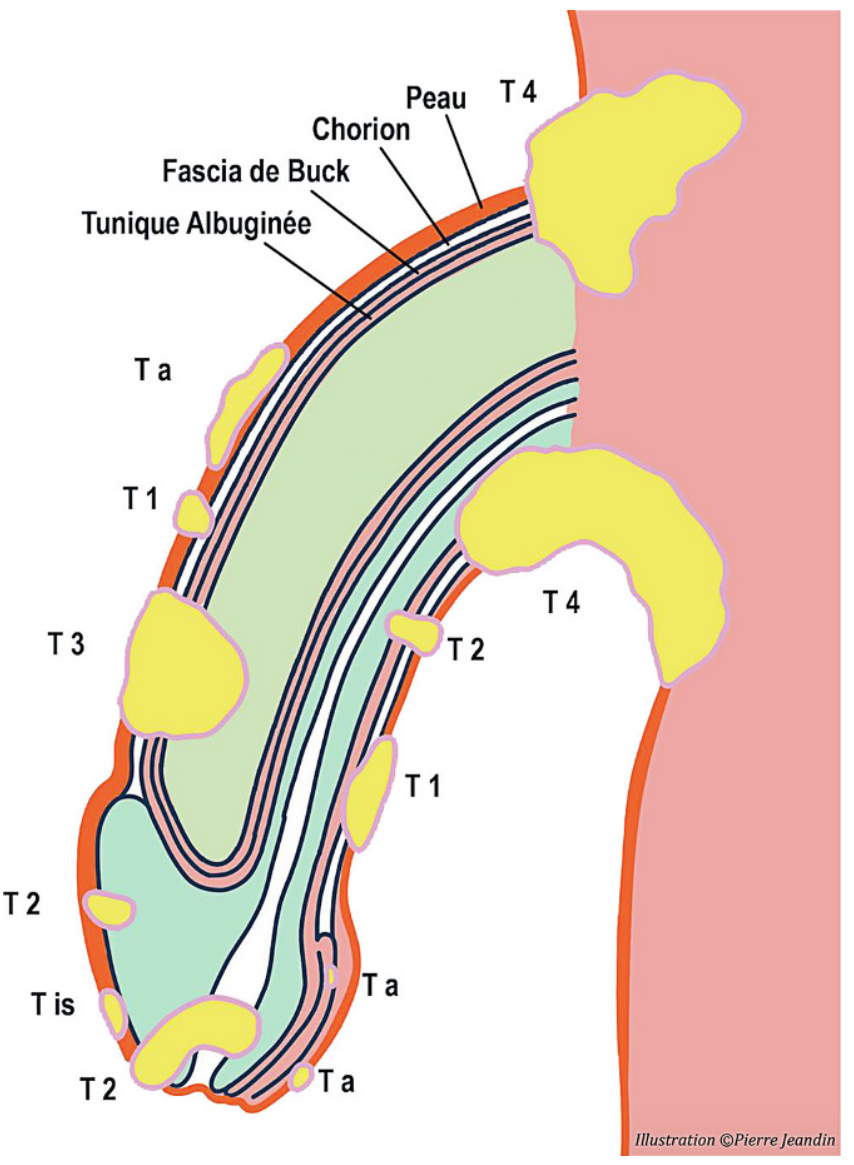

Fig. $1 \varangle$ Stade pathologique du carcinome de la verge seIon la classification TNM (Tumor, Node, Metastasis) 2016. (IIlustration @Pierre Jeandin) douleurs et de nécrose du gland. La radiothérapie externe n'a que peu de place dans le traitement du carcinome épidermoïde de la verge, hormis dans certaines situations palliatives.

En ce qui concerne le traitement des aires ganglionnaires inguinales, la lymphadénectomie inguinale représente le traitement de choix. On compte alors deux techniques:le curage inguinal modifié et la lymphadénectomie inguinale totale. Le curage inguinal modifié consiste en l'ablation des quadrants supérieurs etcentral selon Dasseler, autour de la veine saphène. La lymphade' nectomie inguinale totale consiste quant à elle à l'ablation de tous les ganglions inguinaux du triangle de Scarpa. Elle présente toutefois une morbidité plus élevée, avec des risques non négligeables de lymphocèle, de nécrose cutanée, d'infection pariétale, de lymphorrhée chronique et de lymphœdème, surtout en cas de BMI augmenté. La littérature rapporte une morbidité diminuée lors du port de bas de contention, de l'administration d'une antibiothérapie empirique, de la réalisation de panse-

\section{Pronostic et suivi}

On observe une récidive locale dans environ $15-30 \%$ des cas traités par un geste conservateur, et ce surtout dans les deux premières années post-opératoires, tout particulièrement en cas de marge de résection chirurgicale positive. Le traitement de la récidive locale consiste à nouveau en un geste le plus conservateur possible, alternativement, on procèdera à une amputation partielle ou totale.

La récidive ganglionnaire est traitée par lymphadénectomie inguinale totale, alternativement par radio-chimiothérapie de rattrapage.

La survie spécifique à cinq ans varie fortement selon le stade anatomo-pathologique [6-8]:

- CIS : $100 \%$

- pT1: $84 \%$

- pT2:54\%

- pN0:80-100\%

- $\mathrm{pN}+: 29-40 \%$

- $\mathrm{M}+: 0 \%$

Le suivi est principalement clinique, avec un statut local et des aires ganglionnaires tous les trois mois jusqu'à deux ans, puis tous les six mois jusqu'à cinq ans postopératoires, sauf en cas d'amputation totale ou pour les stades $\mathrm{pN} 0$ où le suivi peut être annuel dès deux ans post-opératoires. Il est vivement recommandé au patient de procéder à des autocontrôles de façon concomitante au suivi médical. Le suivi radiologique demeure optionnel et est plutôt destiné aux stades métastatiques ganglionnaires.

Les conséquences psychologiques du traitement du cancer de la verge sont non négligeables et sont d'autant plus sévères que la mutilation des organes génitaux externes est importante. Certains patients parviennent à obtenir des rapports sexuels satisfaisants selon la longueur résiduelle de la verge, mais la masculinité et l'image corporelle de soi sont souvent dégradées. Il convient ainsi de proposer le cas échéant un accompagnement psychologique à ces patients sur le long terme. 


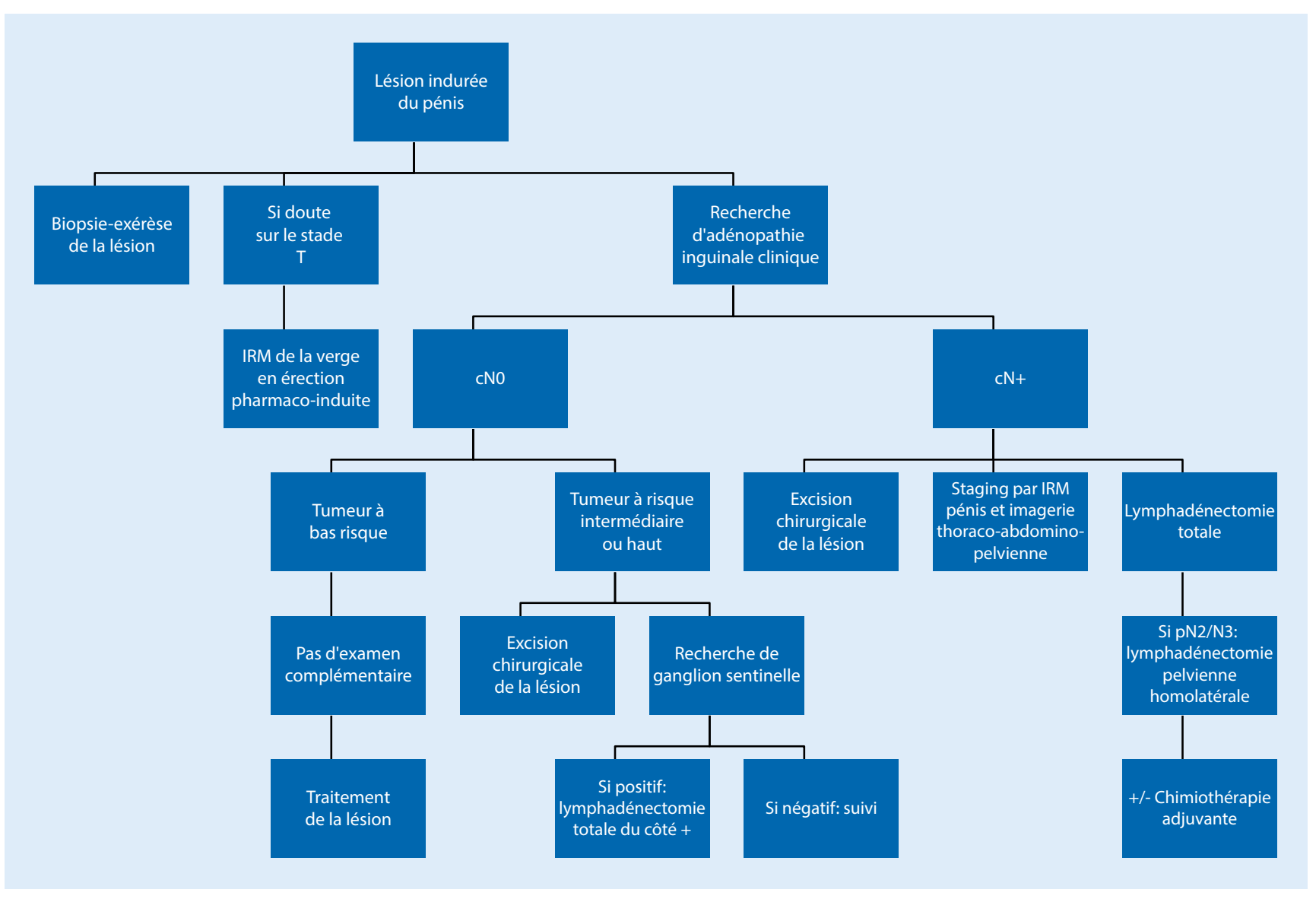

Fig. $2 \Delta$ Algorithme de prise en charge du cancer de la verge selon le stade TNM (Tumor, Node, Metastasis). IRM Imagerie par résonance magnétique, $C N O$ absence de ganglions régionaux inguinaux palpables ou visibles, $C N+$ présence de ganglions régionaux palpables ou visibles, $p N 2$ métastase ganglionnaire dans $>2$ ganglions inguinaux unilatéraux, ou atteinte bilatérale, $p N 3$ métastase ganglionnaire pelvienne, extension extra-capsulaire

\section{Conclusion - Pour la pratique des médecins généralistes}

Le carcinome épidermoïde est le type histologique le plus fréquent des cancers du pénis. Les principaux facteurs de risques identifiés sont les suivants: infections à HPV, phimosis, défaut d'hygiène, inflammation chronique, tabagisme, certains traitements dermatologiques, niveau socio-économique faible, partenaires sexuels multiples, statut célibataire actif sexuellement, premiers rapports sexuels précoces.

L'examen clinique est essentiel pour le staging local et ganglionnaire, puisqu'il guide la réalisation du bilan préopératoire ainsi que la prise en charge chirurgicale. Sélectivement, l'IRM pénienne avec érection pharmaco-induite est l'imagerie de choix. Le traitement du cancer de la verge est essentiellement chirurgical, avec une chirurgie la plus conservatrice possible, et peut être associé à une chimiothérapie en cas d'atteinte métastatique ganglionnaire.

Latteinte ganglionnaire est le facteur pronostic principal et son traitement doit donc être systématique. Il est en principe réalisé, dans la mesure du possible, dans le même temps opératoire que celui de la tumeur initiale.

Un suivi régulier par auto-examination de la verge et des ganglions inguinaux est recommandé, en plus des visites médicales post-opératoires qui sont proposées aux trois mois jusqu'à deux ans puis semestriellement jusqu'à cinq ans post-opératoires.

\section{Adresse de correspondance}

\section{Elisabeth Grobet-Jeandin}

Service d'urologie, Hôpitaux Universitaires de Genève

14 Genève, Suisse

elisabeth.jeandin@hcuge.ch
Elisabeth Grobet-Jeandin médecin interne, Astrid Roosendaal médecin cheffe de clinique

Funding. Open access funding provided by University of Geneva

\section{Conformité aux directives éthiques}

Conflit d'intérêts $E$. Grobet-Jeandin déclare ne pas avoir de conflit d'intérêt.

Pour cet article, les auteurs n'ont réalisé aucune étude sur les humains ou les animaux. Les études réalisées étaient conformes aux normes éthiques indiquées dans chaque cas.

Open Access. This article is licensed under a Creative Commons Attribution 4.0 International License, which permits use, sharing, adaptation, distribution and reproduction in any medium or format, as long as you give appropriate credit to the original author(s) and the source, provide a link to the Creative Commons licence, and indicate if changes were made. The images or other third party material in this article are included in the article's Creative Commons licence, unless indicated otherwise in a credit line to the material. If material is not included in the article's Creative Com- 
mons licence and your intended use is not permitted by statutory regulation or exceeds the permitted use, you will need to obtain permission directly from the copyright holder. To view a copy of this licence, visit http://creativecommons.org/licenses/by/4.0/.

\section{Références}

1. Barnholtz-Sloan JS Maldonado JL, Pow-sang J, Giuliano AR (2007) Incidence trends in primary malignant penile cancer. Urol Oncol 25:361-367. https://doi.org/10.1016/j.urolonc.2006.08.029

2. Hartwig S, Syrjänen S, Dominiak-Felden G, Brotons $M$, Castellsagué $X$ (2012) Estimation of the epidemiological burden of human papillomavirusrelated cancers and non-malignant diseases in men in Europe:a review. BMC Cancer 12:30. https:// doi.org/10.1186/1471-2407-12-30

3. Lont AP, Kroon BK, Horenblas S, Gallee MP, Berkhof J, Meijer CJ, Snijders PJ (2006) Presence of high-risk human papillomavirus DNA in penile carcinoma predicts favorable outcome in survival. Int J Cancer 119:1078-1081. https://doi.org/10.1002/ ijc.21961

4. Perrin A, Iselin CE (2019) Vaccination contre le papillomavirus humain (HPV) chez le sujet masculin: évidence et pratique. Rev Med Suisse 15:2202-2204

5. Maden C, Sherman KJ, Beckmann AM, Hislop TG, Teh CZ, Ashley RL, Daling JR (1993) History of circumcision, medical conditions, and sexual activity and risk of penile cancer. J Natl Cancer Inst 85:19-24. https://doi.org/10.1093/jnci/85.1.19

6. Moses K, Winer A, Sfakianos JP, Poon SA, Kent M, Bernstein M, Russo P, Dalbagni G (2014) Contemporary management of penile can- cer: greater than 15 years MSKCC experience. Can JUro 21(2):7201-7206

7. Veeraterapillary R, Teo L, Asterling S, Greene D (2015) Oncological outcomes of penile cancer treatment at a UK supraregional center. Urology 85(5):1097-1103. https://doi.org/10.1016/j. urology.2014.11.048

8. FossaSDetal(1987) Cancer of the penis. Experience at the Norwegian radium hospital 1974-1985. EurUrol 13(6):372-377

Note de l'éditeur. Springer Nature conserve une position neutre en ce qui concerne les revendications juridictionnelles dans les cartes géographiques et les affiliations institutionnelles figurant dans ses publications.

\section{Gesundheitliche Folgen des Klimawandels: Herausforderung für Nieren und männliche Geschlechtsorgane}

Die Deutsche Gesellschaft für Urologie e.V. (DGU) warnt mit Blick auf wiederkehrende Hitzeperioden vor einer langfristigen Zunahme urologischer Erkrankungen infolge des Klimawandels

„Die gesundheitlichen Folgen häufigerer Extremhitze und höherer Durchschnittstemperaturen beschränken sich nicht allein auf die Zunahme von Schlaganfällen, Herzinfarkten oder Infektionserkrankungen. Sie erhöhen ebenso die Risiken für urologische Erkrankungen, von Harnsteinen bis hin zu schweren Nierenschädigungen", sagt Prof. Jens Rassweiler, Präsident der DGU.

Im urologischen Alltag werden verschiedene klimasensible Erkrankungen vermehrt auftreten. „Durch hitzebedingte Austrocknung des Körpers wird der Wasserund Blutsalz-Haushalt beeinträchtigt und führt zur Abnahme der Nierenfunktion. Die daraus folgende Volumendepletion und Hyperosmolarität begünstigen häufigere Steinbildungen", sagt Prof. Joachim Steffens. Als weitere Auswirkung hält der Chefarzt der Klinik für Urologie und Kinderurologie am St.-Antonius-Hospital in Eschweiler auch eine Beeinträchtigung der männlichen Zeugungsfähigkeit für denkbar.

Im Fokus der Urologen stehen temperaturbedingte Nierenerkrankungen. „Die Nieren spielen eine zentrale Rolle beim Schutz des Menschen vor hitzebedingten Folgen, sind zugleich aber auch Zielorgan hitzeassoziierter Schäden. Das Spektrum reicht von der akuten Nierenschädigung, über eine erhöhte Inzidenz von Nierensteinen und Harnwegsinfekten bis hin zur chronischen Nierenschädigung", so Prof. Ralph Kettritz, Facharzt für Innere Medizin und Nephrologie an der Charité - Universitätsmedizin Berlin.

Für die Nieren sei es eine große Herausforderung, unter extremer Hitze Elektrolyte und Wasser im Körper zu konservieren und bezüglich Menge und Konzentration in einem Gleichgewichtszustand zu halten. Gelinge dies nicht, verliere der Körper wichtige Stoffe und der renale Blutfluss nehme ab. Dazu kommt eine maximale Stimulation des antidiuretischen Hormons (ADH), um Wasser zu konservieren. Diese Mechanismen seien an der
Entstehung einer akuten Nierenschädigung beteiligt. „Die Wasserkonservierung wird über eine maximale Konzentrierung des Urins durch ADH erreicht. Bei reduziertem Urinfluss werden aufsteigende Infektionen im Harntrakt begünstigt. Darüber hinaus wird im hochkonzentrierten Urin die Steinbildung gefördert", sagt Prof. Kettritz.

Auch die Fragestellung nach einem Zusammenhang zwischen steigenden Temperaturen und einer Zunahme der Infertilität unter Männern der nördlichen Regionen beschäftigt die Urologen. Prof. Steffens: „Eine erhöhte Umgebungstemperatur der Hoden vermindert die Spermienqualität und kann dadurch die männliche Zeugungsfähigkeit beeinträchtigen. Die Evolution hat die Hoden des Mannes außerhalb der Körperhöhle platziert, da die dort zwei bis drei Grad niedrigere Temperatur gegenüber der Körperkerntemperatur von $37^{\circ} \mathrm{C}$ zu einer guten Samenqualität führt." Bekanntermaßen erhöhe etwa ein Krampfaderbruch die Temperatur im Hodensack und begünstige damit nachweislich die Zeugungsunfähigkeit. Dieses temperaturbedingte Krankheitsgeschehen lasse sich möglicherweise auch auf eine temperaturbedingte Steigerung der Infertilität als Folge des Klimawandels übertragen. Wissenschaftliche Daten gebe es dazu bislang jedoch nicht.

Prof. Steffens sieht deshalb dringenden Bedarf an entsprechenden wissenschaftlichen Erkenntnissen: „Es gibt noch keine systematische Forschung zu Einflüssen der Erderwärmung auf Erkrankungen des Harntraktes und der männlichen Geschlechtsorgane". Er regt daher die Bildung interdisziplinärer Forschungsverbünde wie etwa mit der Nephrologie an, um Wissen über die urologischen Folgen des Klimawandels zu generieren.

Quelle: Bettina-Cathrin Wahlers, Pressestelle der DGU 